\title{
PROBLEMAS Y PERSPECTIVAS DE LA UNIVERSIDAD PERUANA CASO: INGENIERÍA ELECTRÓNICA - UNPRG - LAMBAYEQUE UN ENFOQUE DE LOS SISTEMAS VIABLES
}

\author{
PROBLEMS AND PROSPECTS OF THE PERUVIAN UNIVERSITY \\ CASE: ELECTRONIC ENGINEERING - UNPRG - LAMBAYEQUE \\ A VIABLE SYSTEMS APPROACH
}

\section{JULIO ERNESTO QUISPE ROJAS', JULIO ENRIQUE QUISPE TUESTA ${ }^{2}$}

1 Universidad Nacional Pedro Ruiz Gallo, Lambayeque (Perú).jquisperojas@gmail.com

2 Universidad Señor de Sipán, Chiclayo (Perú).julioqt@gmail.com

\begin{abstract}
RESUMEN
La investigación sobre los problemas y perspectivas de la Universidad Peruana, tiene por finalidad identificar los procesos que afectan al desarrollo de la universidad, que no se adecua a los tiempos actuales donde la globalización exige niveles de calidad en la formación profesional, en las investigaciones y en la gestión misma de la universidad, y donde las tecnologías de la información son un soporte determinante para la competitividad de las organizaciones. Se planteó seleccionar una universidad y una carrera profesional relacionada con la actividad principal de la región Lambayeque, analizar su vínculo con la investigación, el desarrollo tecnológico y la innovación, y utilizar el enfoque de sistemas que permite analizar los elementos y las relaciones entre ellos, la cibernética organizacional y específicamente el modelo de los sistemas viables de Stafford Beer, que privilegia las relaciones con el entorno y las tecnologías de la información como soporte para la buena gestión de la organización. Se levantó la información mediante un cuestionario con preguntas cerradas y abiertas sobre los problemas y las propuestas de solución a 74 docentes de 31 universidades de todas las regiones del país, y se validó la existencia de los problemas en un caso de estudio, en la Escuela Profesional de Ingeniería Electrónica de la Universidad Nacional Pedro Ruiz Gallo de Lambayeque (Perú).
\end{abstract}

PALABRAS CLAVE: universidad peruana, modelo de sistemas viables, cibernética organizacional.
Research on the problems and prospects of the Peruvian University, aims to identify problems affecting the development of the university, which is not suited to the times where globalization requires levels of quality in vocational training, research and in the same university management, and where information technologies are a key support for organizational competitiveness. It was proposed to select a university and a professional specialty related to the main activity in the region, analyzing their links research, technological development and innovation, and use the systems approach, organizational cybernetics and specifically the viable systems model Stafford Beer, which promotes relations with the environment and information technology as support for good governance of the organization. Information is lifted using a format on the problems and proposed solutions to 74 teachers from 31 universities from all regions of the country, and validated the existence of problems in a case study in the Professional School of Electronic Engineering University National Pedro Ruiz Gallo, Lambayeque (Peru).

KEYWORDS: peruvian university, viable systems model, organizational cybernetics. 
INTRODUCCIÓN

El norte del Perú es una región dedicada a la agricultura, tradicionalmente ha tenido como producción agrícola fundamental el cultivo de la caña de azúcar, seguido del arroz, industrializándolos para el consumo interno y excepcionalmente para la exportación. Actualmente otros productos agrícolas puestos en los mercados internacionales han despertado la preferencia de los consumidores, y se ha generado una modificación en las actividades, sembrándose frijoles, ajíes, pimientos, espárragos, frutas, entre otros para la exportación.

El escenario muestra una situación heterogénea. Por ejemplo, mientras la industria azucarera funciona con tecnologías antiguas, los molinos de arroz poseen una tecnología moderna que incluye la selección por color laser. Las empresas agroindustriales dedicadas a la exportación, son de reciente formación y su equipamiento es de tecnología moderna para cumplir con las exigencias de calidad y precio que les permitan ubicarse en el mercado internacional.

Las empresas de los países desarrollados y países emergentes cuentan con sistemas de investigación, desarrollo tecnológico e innovación $(\mathrm{I}+\mathrm{D}+\mathrm{I})$, muy articulados con su entorno, que vinculan las universidades, las empresas y los organismos del gobierno, situación que no se presenta en el Perú. Las universidades deben cumplir con sus funciones sustantivas de enseñanza aprendizaje, investigación, extensión universitaria y proyección social, y cumplir con los niveles de calidad que exigen el mundo competitivo actual.

\section{ANTECEDENTES}

En un estudio realizado por uno de los autores, en el 2009, sobre las innovaciones en las empresas industriales de Lambayeque, se determinó que un $14 \%$ de los supervisores entrevistados, percibía al Concejo Nacional de Ciencia, Tecnología e Innovación Tecnológica (CONCYTEC) como una institución que apoya y promueve la innovación, seguido por el Servicio Nacional de Adiestramiento en Trabajo Industrial (SENATI), con un $11 \%$, luego siguen las universidades con un 6\%, la Cámara de Comercio y Producción de Lambayeque con 6\%, Comisión para la Promoción de Exportaciones del Perú (PROMPEX) y Organismos No Gubernamentales también cada uno con el 6\%, la Asociación de Exportadores (ADEX) 3\%, el Ministerio de Agricultura
3\%, Ministerio de Comercio Exterior y Turismo (MINCETUR) 3\%, Sociedad Nacional de Industrias 3\%, las mismas empresas se financian y apoyan en un $30 \%$ y otras empresas industriales $9 \%$ (Quispe, 2011).

En el mencionado estudio, determinó que el $40 \%$ de las innovaciones estudiadas fueron referentes al mejoramiento del proceso productivo; el $29 \%$ relacionada con el producto; el $21 \%$ en ambos (proceso y producto); y, un $10 \%$, estaba referido a innovaciones en el proceso de distribución, métodos de venta, formas de pago, etc. Otro de los hallazgos fue que sólo el 3\% de las empresas estudiadas realizaban un esfuerzo consciente por mejorar su competitividad, el 74\% de las empresas hacía algún tipo de esfuerzo y el $23 \%$ no realizaba ninguno.

UNIVERSIDADES

En el Perú las universidades se rigen por la Ley Universitaria No. 23733, de diciembre de 1983, promulgada por el presidente Fernando Belaunde Terry y complementada por el Decreto Legislativo No. 882 de noviembre de 1996, del presidente Alberto Fujimori. Se consideran tres tipos de universidades: las públicas, que brindan la formación profesional gratuita y reciben la asignación presupuestal del gobierno; las privadas asociativas (sin fines de lucro), cuyos alumnos tienen que pagar una pensión de estudios y sus utilidades deben dirigirse a su desarrollo, pues los promotores no tienen la propiedad de ella, si no la comunidad universitaria; y las universidades societarias que tienen dueños como las empresas privadas y pueden disponer de sus utilidades (tabla 1).

\section{TABLA 1. NÚMERO DE UNIVERSIDADES POR TIPO}

\begin{tabular}{lr}
\hline TIPO DE UNIVERSIDAD & UNIVERSIDADES \\
\hline Pública & 31 \\
Privada Asociativa & 19 \\
Privada Societaria & 25 \\
Total & 75 \\
\hline
\end{tabular}

Fuente: los autores.

Perú se puede dividir en tres regiones por su ubicación geográfica: Región Norte, que comprende nueve departamentos; Región Centro, con siete departamentos; y la Región Sur, con ocho departamentos. Lima, la capital del Perú, y la Provincia Constitucional del Callao tienen una población mayor a nueve millones y Perú tiene una población de treinta millones, y forman una región. 
Para la clasificación de las universidades por ubicación geográfica se han tomado cuatro áreas geográficas (figura 1 y tabla 2).

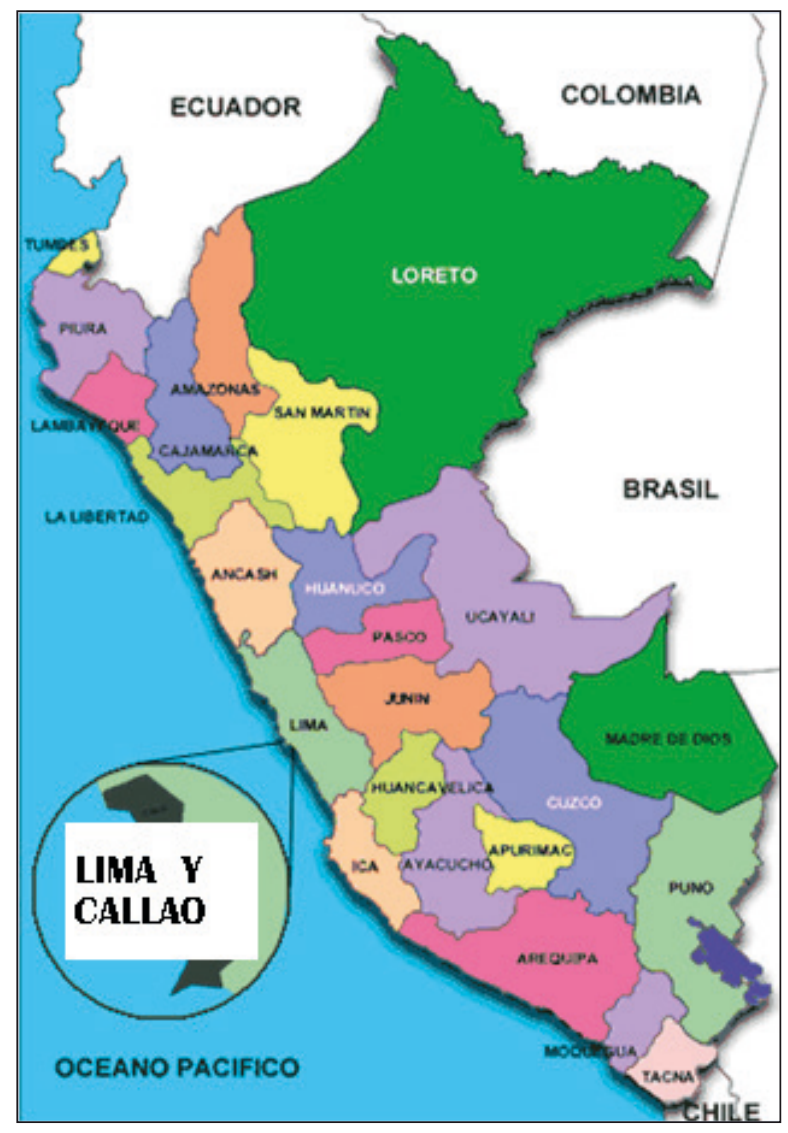

Figura 1. Mapa político de Perú

Fuente: I.E.E. Honorio Delgado, 2013- Adaptación.

El Perú, para el año 2013 tenía institucionalizadas setenta y cinco universidades. En la tabla 2 se muestran las universidades por cada región, considerando la capital como una región, que incluye Lima y Callao.

\section{TABLA 2. NÚMERO DE UNIVERSIDADES POR REGIÓN}

\section{REGIÓN}

UNIVERSIDADES

Norte

Centro 10

Sur $\quad 16$

Capital

Total

75

Fuente: Elaboración propia basada en Estadística de la Asociación Nacional de Rectores del Perú (ANR, 2011).

ENFOQUE DE SISTEMAS

El enfoque de sistemas en el análisis de las organizaciones no centra la atención en las partes sino en las relaciones entre las partes y las rela- ciones de ellas con el entorno, destacando que hay propiedades del todo que no posee ninguna de las partes.

Este enfoque tiene dos influencias metodológicas importantes: la analítica, que permite describir el objeto de estudio, y la sintética que permite comprender la función o razón de ser del objeto de estudio. Así, el enfoque sistémico para diseñar un nuevo sistema o mejorar uno existente, primero debe identificar el sistema superior que lo contiene para después definir cuál va a ser la función que cumplirá el objeto de estudio en el sistema superior.

La Cibernética Organizacional (CO) se enmarca dentro del pensamiento sistémico. La cibernética, se define como la ciencia de la comunicación y el control en los seres vivos y las máquinas. Por su parte, la cibernética organizacional aplica sus principios al estudio de las organizaciones.

El Stafford Beer con la publicación de $\mathrm{Cy}$ bernetics and Management en 1959, contribuyó al nacimiento de la CO. En esta obra plantea la aplicación de los principios de la ciencia cibernética al estudio de las organizaciones. En 1972 Beer propuso el Modelo de los Sistemas Viables (MSV) en su libro Brain of the Firm, y luego en 1979 con The Heart of Enterprice completa los fundamentos teóricos del modelo de los sistemas viables. En 1985 con Diagnosing the System for Organization, da las pautas metodológicas para la aplicación en el diagnóstico o diseño organizacional.

Leonid Ototsky (2006), jefe del Sistema de Gestión e Integración del Moscow Institute of Physics and Technology (MIPT) de Moscú, en su investigación Stafford Beer y el Sistema Viable en el siglo XXI, plantea que la complejidad y las actuales capacidades y desarrollos del Internet, están sobrepasando el alcance visionado por sus creadores, y comenta que las potencias en ventas de Enterprice Resource Planning (ERP), como SAP y Oracle, están creando fuertes herramientas analíticas para apoyar la viabilidad de las empresas, algunos ejemplos son la supervisión de actividades económicas de Oracle y el gestor estratégico de empresas de SAP.

Pérez Ríos (2008) actualiza la importancia del modelo de los sistemas viables, legado de Stafford Beer, con su propuesta metodológica Diseño y diagnóstico de organizaciones viables, un enfoque sistémico complementado con el software VSMod para facilitar la aplicación de la cibernética organizacional en general y del modelo de sistemas viables en especial. 
METODOLOGÍA PREGUNTAS Y OBJETIVOS

La universidad peruana está regida por la Ley 23733 de 1983, y no se ha adecuado a las tendencias mundiales en la formación profesional, teniendo una serie de problemas que impiden a la universidad lograr una cultura de la calidad y de excelencia académica. El análisis de la realidad de la universidad peruana, que realizan estudiosos del tema y los equipos de las autoridades responsables de la formación profesional en el Perú, es llevado a cabo con enfoques tradicionales. Estos, no permiten determinar correctamente las perspectivas de la universidad, en tiempos actuales de globalización, de las tecnologías de la información, de la internacionalización de las universidades, de la importancia de la calidad, de la existencia de redes de investigación y las acciones conjuntas de las universidades con las empresas y los gobiernos.

Ante este contexto, surgen dos preguntas: ¿cuáles son los problemas de la universidad peruana? y ¿cuáles son las perspectivas de la universidad peruana?, que respondemos en el presente trabajo.

El objetivo general de la investigación es plantear las perspectivas de la universidad peruana desde el enfoque de los sistemas viables en un caso de estudio.

Los objetivos específicos son:

- Seleccionar una carrera profesional de una universidad pública, un sector de la actividad económica y un campo de acción para tomar como caso de estudio.

- Identificar los problemas generales de las universidades del Perú y las propuestas de solución a esos problemas, y validarlos en el caso de estudio.

- Analizar la carrera profesional en estudio desde el enfoque de los sistemas viables, y determinar las perspectivas de la universidad peruana.

\section{ELECCIÓN DE CASO DE ESTUDIO}

La selección de la carrera profesional para el caso de estudio debe ser un centro de estudios representativo de la universidad peruana, con una carrera profesional que esté relacionada con la actividad económica principal de la región donde está enclavada la institución y con algún campo de acción que contribuya a la mejora de la calidad de vida de la sociedad.

El enfoque para el análisis debe privilegiar las nuevas tendencias para la organización y direc- ción de las instituciones y empresas, haciendo posible el análisis de las nuevas variables de un mundo cambiante y altamente competitivo como el actual. En el caso de estudio se deben verificar los problemas planteados y analizar las perspectivas teniendo en cuenta el enfoque seleccionado, que es el enfoque de los sistemas viables.

\section{PROBLEMAS Y PROPUESTAS DE SOLUCIÓN}

Para identificar los problemas generales de la universidad peruana, se elaboró un formato de entrevista a docentes de diferentes categorías, tipos de universidad y áreas geográficas del Perú, documento que incluye datos generales del entrevistado, y dos pedidos. El primero: indicar dos de los problemas principales de la universidad peruana, y el segundo: indicar dos propuestas para mejorar la universidad peruana.

\section{POBLACIÓN Y MUESTRA}

La población estuvo formada por todas las universidades del Perú. La muestra se realizó por conveniencia, en base a la relación de los autores con los docentes de las universidades del Perú, contactos que se establecieron en 10 años de participación en diversos cursos, conferencias y congresos realizados por la Asamblea Nacional de Rectores del Perú. Se planteó la necesidad de contar con, por lo menos, dos opiniones por cada universidad. Después de validar, la muestra estuvo compuesta por setenta y cuatro docentes de treinta y uno universidades.

Se entrevistó a docentes universitarios de los tres tipos de universidades, en la tabla 3 se muestra la cantidad de universidades, según tipo, y el número de docentes que brindaron información para la investigación.

\begin{tabular}{lrr} 
TABLA 3. UNIVERSIDADES Y DOCENTES ENTREVISTADOS POR TIPO \\
DE UNIVERSIDAD & \\
\hline TIPO DE UNIVERSIDAD & UNIVERSIDADES & DOCENTES \\
\hline Universidad Pública & 16 & 38 \\
Universidad Privada Asociativa & 9 & 18 \\
\hline Universidad Privada Societaria & 7 & 18 \\
\hline Total & 31 & 47 \\
\hline
\end{tabular}

Fuente: los autores.

Los docentes entrevistados pertenecen a universidades de las cuatro regiones consideradas y en la tabla 4, se muestran las cantidades por cada región.

En Perú, los docentes universitarios se pueden clasificar en tres categorías. Los docentes de categoría auxiliar, son los que ingresan a la 
TABLA 4. UNIVERSIDADES Y DOCENTES ENTREVISTADOS POR REGIÓN

\begin{tabular}{lrr}
\hline REGIÓN & UNIVERSIDADES & DOCENTES \\
\hline Región Norte & 12 & 36 \\
\hline Región Centro & 7 & 14 \\
\hline Región Sur & 5 & 10 \\
Capital & 7 & 14 \\
TOTAL & 31 & 74 \\
\hline
\end{tabular}

Fuente: los autores.

docencia universitaria con el requisito de tener el título profesional y estar inscrito en el colegio profesional correspondiente, después de tres años pueden ascender a la categoría de asociado, cumpliendo requisitos de investigación, grados académicos y capacitación, entre otros. Después de cinco años en la categoría, el asociado puede ascender a la categoría principal, quienes son los docentes de mayor experiencia profesional y de la vida universitaria.

Los docentes de categoría principal pueden ser: a dedicación exclusiva, cuando se dedican solo al trabajo en la universidad en forma exclusiva, no pudiendo recibir beneficios económicos de otras entidades, y los de tiempo completo que deben trabajar las cuarenta horas semanales y pueden realizar otras actividades económicas en las demás horas del día y de la semana (tabla 5).

TABLA 5. DOCENTES ENTREVISTADOS POR CATEGORÍA

\begin{tabular}{lr}
\hline CATEGORíA & DOCENTES \\
\hline Principal a dedicación exclusiva & 7 \\
\hline Principal a tiempo completo & 34 \\
\hline Asociado & 24 \\
\hline Auxiliar & 9 \\
Total & 74 \\
\hline
\end{tabular}

Fuente: los autores.

LEVANTAMIENTO Y ANÁLISIS DE LOS DATOS

El levantamiento de la información se realizó mediante entrevista directa, vía telefónica, vía Skype y vía correo electrónico, utilizando para ello los formatos antes descritos. Estos fueron llenados por los entrevistados en algunos casos y en otros por el entrevistador, cuando la entrevista no era directa.

Las respuestas de los setenta y cuatro docentes que apoyaron con sus opiniones al presente estudio, se agruparon en dos tablas. La primera sobre los problemas de las universidades peruanas y la segunda sobre las propuestas de solución a esos problemas. Luego estos problemas, para ser validados como tales, fueron verificados en el caso de estudio, identificándose las evidencias que confirman su existencia en el caso.

\section{RESULTADOS \\ INGENIERÍA ELECTRÓNICA DE LA UNIVERSIDAD NACIONAL PEDRO RUIZ GALLO, AGROINDUSTRIA E I+D+I}

Se ha tomado para la presente investigación el caso de la Escuela Profesional de Ingeniería Electrónica de la Universidad Nacional Pedro Ruiz Gallo de Lambayeque, Perú, y el sector agroindustrial de la Región Lambayeque y su vínculo en la investigación, el desarrollo tecnológico y la innovación para la automatización de las plantas industriales de las empresas del sector.

La Escuela Profesional de Ingeniería Electrónica inicia sus actividades en abril del año 2000. Para el año 2013 tenía 390 alumnos y 385 egresados, de los cuales 183 están titulados.

La actividad principal de Lambayeque es la agricultura, básicamente el cultivo de la caña de azúcar y arroz. No obstante, en la última década se ha incursionado en la agroindustria de exportación, compuesto por frutas, ajíes, espárragos y frijoles. Las plantas de procesamiento están en pleno desarrollo, tanto por las mejoras realizadas por los mismos técnicos y operarios de las empresas, como por transferencia de tecnología mediante compra de equipos y capacitación del personal.

El vínculo de la universidad con las empresas y el gobierno en el tema de la investigación, el desarrollo tecnológico y la innovación, es una competencia que deben desarrollar los futuros profesionales y los miembros de las empresas. Esta es la razón por la que se elige este campo de acción.

PROBLEMAS DE LA UNIVERSIDAD PERUANA

Los problemas agrupados se sometieron a su validación en el caso de estudio, para ello se realizó el análisis documentario de la Escuela Profesional de Ingeniería Electrónica y se entrevistó a los docentes que desempeñaron el cargo de Directores de Escuela en los catorce años de funcionamiento, así como a tres docentes que tienen cátedra en la carrera profesional de Ingeniería Electrónica.

Los problemas validados en el caso de estudio con las evidencias, se presentan como una generalización de los problemas de la Universidad Peruana, en base a lo identificado en el caso de estudio. Los problemas seleccionados son siete y se muestran en la tabla 6 . 
TABLA 6. PROBLEMAS Y SUS EVIDENCIAS EN EL CASO DE ESTUDIO

\begin{tabular}{|c|c|c|}
\hline NO. & PROBLEMA & EVIDENCIAS \\
\hline 1 & $\begin{array}{l}\text { La universidad está aislada de la realidad } \\
\text { que la rodea }\end{array}$ & $\begin{array}{l}\text { - No hay prácticas para los alumnos formalizadas mediante convenios con las asociaciones de empresarios. } \\
\text { - No hay comité consultivo de la carrera. } \\
\text { - Los docentes no participan en pasantías y asistencia técnica a las empresas. } \\
\text { - Inexistencia de flujo de recursos económicos de las empresas a la universidad. } \\
\text { - Escaso conocimiento de las actividades que realizan las empresas donde piensan trabajar los futuros } \\
\text { profesionales }\end{array}$ \\
\hline 3 & $\begin{array}{l}\text { Carencia de un sistemas de medición, } \\
\text { supervisión y control de las actividades de } \\
\text { la universidad }\end{array}$ & $\begin{array}{l}\text { - No se dispone de información sobre los resultados de las evaluaciones en forma global. } \\
\text { - No se tienen datos consolidados de los resultados de las investigaciones. } \\
\text { - No hay información completa sobre las acciones de Extensión universitaria y proyección social. } \\
\text { - Falta de propuestas para una gestión universitaria basada en los principios fundamentales de mejora } \\
\text { continua. }\end{array}$ \\
\hline 5 & $\begin{array}{l}\text { Carencia de competencias para la gestión } \\
\text { universitaria en directivos, docentes y } \\
\text { administrativos }\end{array}$ & $\begin{array}{l}\text { - No se tiene una cultura de planeamiento de la gestión a todo nivel. } \\
\text { - Lentitud en la toma de decisiones. } \\
\text { - Desconocimiento de la normatividad. } \\
\text { - Falta de previsión de los recursos económicos para la ejecución de las actividades e inversiones para la } \\
\text { mejora de la formación profesional. } \\
\text { - Accionar reactivo del aparato administrativo. }\end{array}$ \\
\hline 7 & Deficiente gestión de la información. & $\begin{array}{l}\text { - Información parcial, incompleta y no oportuna. } \\
\text { - Procesos manuales que retardan la gestión y el cumplimiento de las metas. } \\
\text { - Falta de información consolidada para la toma de decisiones. } \\
\text { - Duplicidad de información e incoherencias, con datos diferentes y datos faltantes. } \\
\text { - Falta de un tablero o cuadro integral de mando para supervisar el cumplimiento de lo planificado. }\end{array}$ \\
\hline
\end{tabular}

Fuente: los autores.

\section{PROPUESTAS DE SOLUCIÓN}

La tabla de propuestas de solución elaborada en base a las respuestas de los docentes de la muestra, se sometió al análisis de pertinencia de los problemas planteados y después de agregarle las acciones para la ejecución de las propuestas, se pidió la opinión del director actual de la Escuela Profesional, exdirectores y los docentes participantes.

La tabla de propuestas y sus acciones para su ejecución se tendrá en cuenta para el análisis de las perspectivas de la carrera profesional desde el enfoque de los sistemas viables (tabla 7).

\section{DISCUSIÓN \\ MODELO DE LOS SISTEMAS VIABLES}

El Modelo de los Sistemas Viables (MSV), está conformado por cinco sistemas (figura 2):

- Sistema 5: sistema que involucra la cultura organizacional y los valores de los directivos.

- Sistema 4: sistema relacionado con la planificación y dedicado al manejo del futuro y el entorno.

- Sistema 3: sistema que se ocupa de las actividades administrativas relacionadas con el corto plazo y el ambiente interno.

- Sistema 2: sistema de regulaciones que comprende las normas, procedimientos y los estándares de gestión.

- Sistema 1: sistema que se dedica a las actividades operativas de la organización. 


\section{TABLA 7. PROPUESTAS DE SOLUCIÓN Y ACCIONES A EJECUTAR}

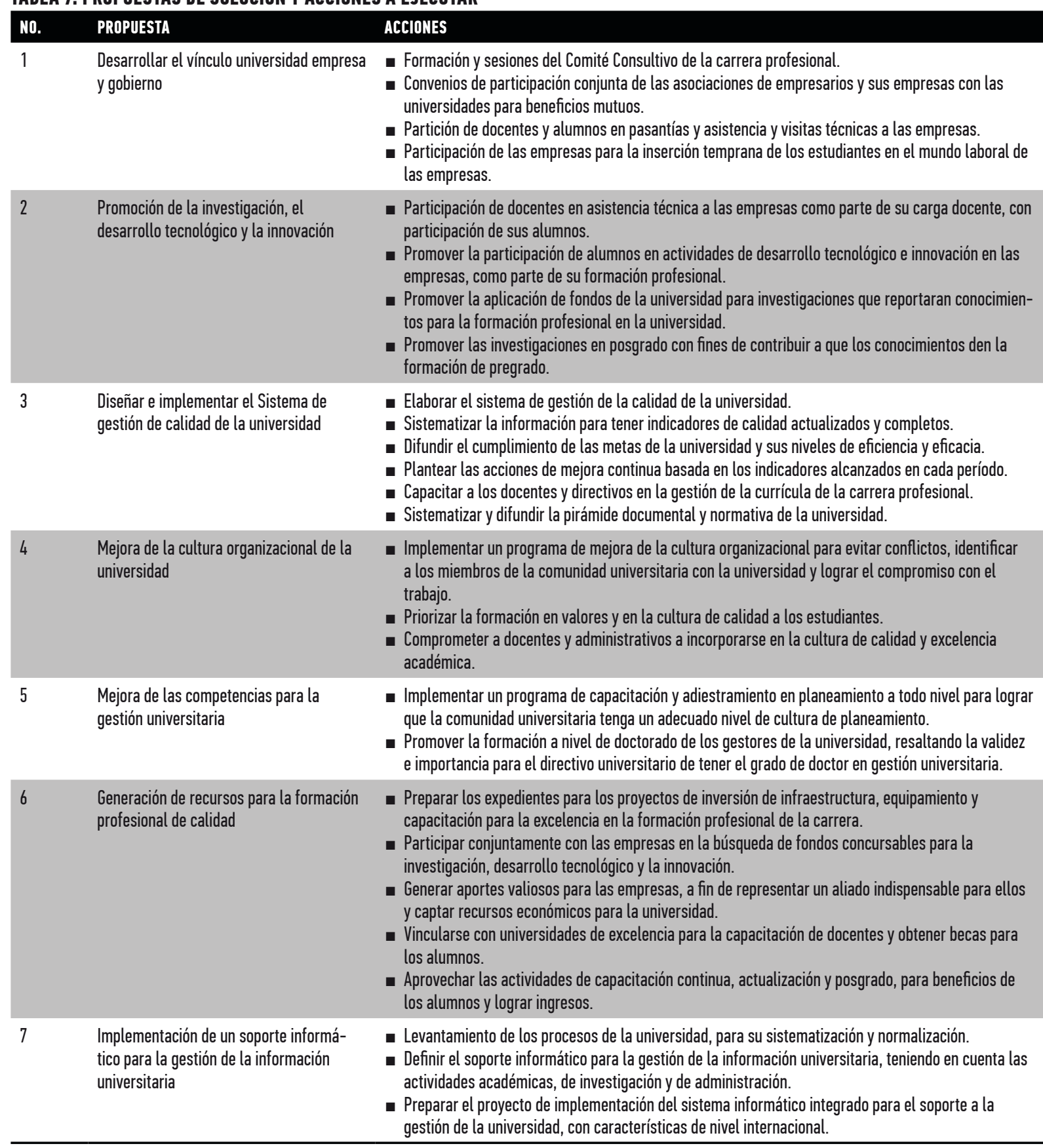

Fuente: los autores.

\section{RELACIONES OPERACIONES ENTORNO}

El modelo de los sistemas viables prioriza las relaciones de la organización con el entorno, como se puede notar en el modelo, se muestran las relaciones de cada una de las áreas de operaciones de la organización con su correspondiente entorno específico, así las relaciones de las escuelas profesionales y de los docentes miembros de la escuela con su entorno cercano debe ser evidente. En el caso de estudio, los vínculos de los docentes de los cursos de automatización industrial con las empresas y los supervisores de las empresas agroindustriales debe ser intensa para poder contribuir al beneficio mutuo, en el caso de los docentes de sistemas telefónicos con los trabajadores de las empresas de telecomunicaciones, también debe ser resaltado. Identificar para cada área operativa de la organización su correspondiente entorno, por cercanía y pertinencia, también se conoce como desdoblamiento vertical en el modelo de los sistemas viables. 


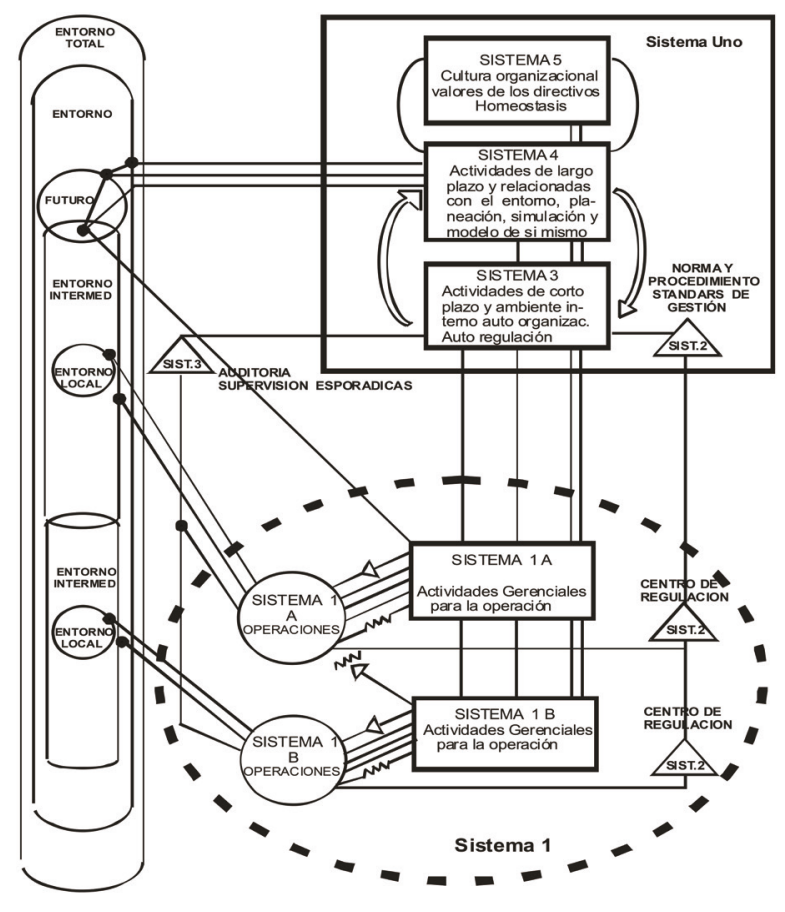

Figura 2. Modelo de los sistemas viables Fuente: Beer, 1985

El manejo de las relaciones UniversidadEmpresa-Gobierno se basará en la Ley de Ashbi que dice: "solo la variedad puede absorber la variedad", significa que la variedad generada por el entorno solo puede ser enfrentada por la variedad generada por la organización. Si el desbalance de variedad, como es natural, es a favor del entorno, entonces el modelo de gestión debe tener mecanismos para compensar y lograr el equilibrio. Si es posible superar la variedad del entorno y lograr una organización competitiva, una universidad puede ser reconocida por su calidad y aporte.

\section{COMPENSADORES DE VARIEDAD}

Para valorar el grado de complejidad de una situación o problema, se utiliza el concepto de variedad, que equivale al número de estados posible y comportamientos actuales o potenciales que se pueden dar en una determinada situación o problema; y no se trata de medir con precisión su valor, sino de reflejar la dimensión del problema al que se enfrentan los directivos al dirigir la organización (figura 3).

El Modelo de Beer propone compensadores de variedad, uno en sentido de mayor variedad a menor variedad llamado atenuador y otro en sentido de menor variedad a mayor variedad llamado amplificador. El uso del atenuador y el amplificador también se conoce con desdoblamiento horizontal.

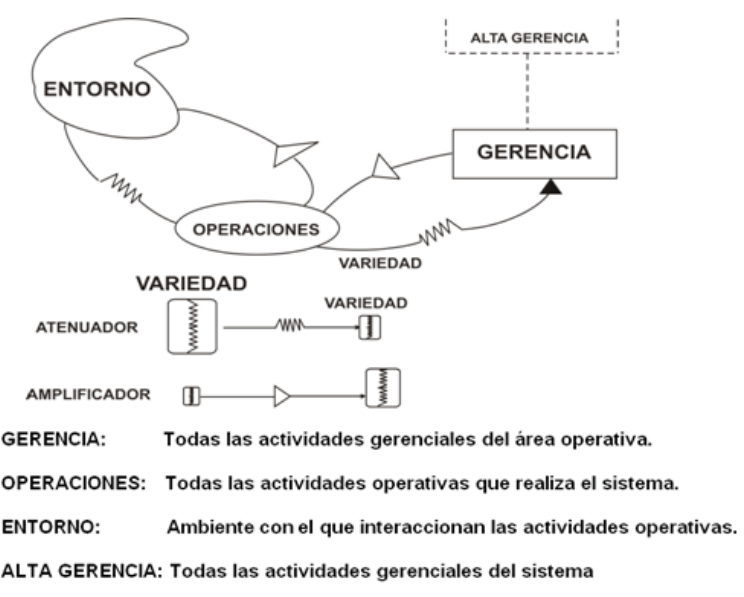

Figura 3. Compensadores de variedad Fuente: Beer, 1985

El atenuador reduce la variedad, esto es, convierte la alta variedad de la entrada en baja variedad a la salida, permiten seleccionar o filtrar de la gran variedad existente en el entorno solo las situaciones pertinentes a la organización, y que debe hacer frente para mantener su viabilidad.

Los atenuadores también permiten que por excepción, los niveles superiores de la organización atiendan a los requerimientos del entorno, mediante la estandarización de los procedimientos en todos los niveles de la organización, utilizando por ejemplo estudios de investigación para determinar el mercado objetivo de una determinada actividad, donde la atenuación reduce todo el mercado al mercado objetivo producto de la investigación de mercado.

El amplificador cumple la función inversa, amplifica la variedad, convierte la baja variedad de la entrada en alta variedad a la salida, permiten aumentar la variedad de la organización mediante el despliegue de mayor capacidad frente al entorno, por ejemplo mediante el uso de las redes sociales, dando a conocer las características de los servicios y productos que la organización brinda a la sociedad.

Con ello la limitada atención que puede brindar una ventanilla de atención al público se amplía considerablemente con el uso de las tecnologías de la información, que oriente y facilite el actuar ordenado y oportuno de los usuarios ante las instancias adecuadas. En el caso de estudio, las comunicaciones a las empresas agroindustriales mediante cartas, afiches, calendarios de la Escuela Profesional de Ingeniería Electrónica, mantiene en vigencia la relación y promociona sus servicios y a los futuros profesionales que egresaran. 
PLANEACIÓN Y TOMA DE DECISIONES

El Modelo de Beer considera a través del Sistema 4 la planeación, el futuro y el entorno como un componente importante que le permite tener una referencia de sí mismo y analizar mediante la simulación los efectos de las estrategias y decisiones; y así mismo, evaluarlas y realizar mediante la retroalimentación las variantes necesarias para responder a los cambios del entorno y del ambiente interno. Los desarrollos tecnológicos actuales brindan a las organizaciones un soporte para la gestión que constituye ventaja competitiva para su éxito y las universidades deben al igual que las empresas realizar un alineamiento estratégico de las tecnologías de la información con las estrategias de desarrollo (figura 4).

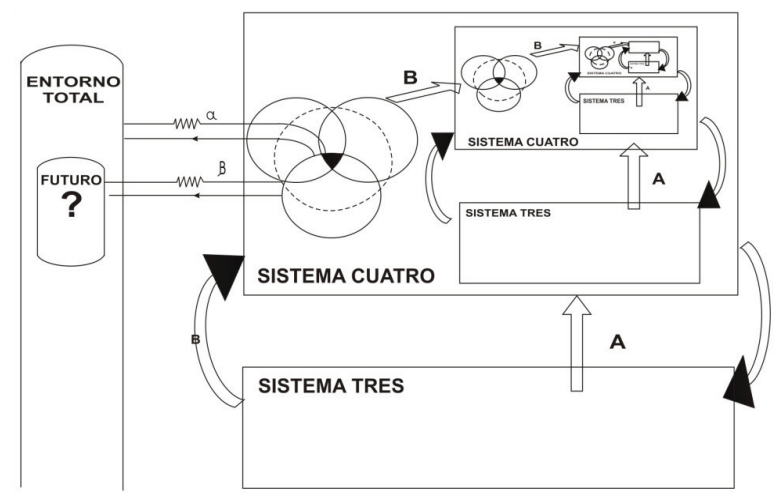

Figura 4. Simulación, control y toma de decisiones Fuente: Beer, 1985.

Las perspectivas de la Universidad Peruana es ser una universidad de calidad, con investigaciones que contribuyan a la competitividad de las empresas y a la sostenibilidad del planeta, con un manejo adecuado de la información para decisiones oportunas y acertadas, realizando un alineamiento estratégico de las tecnologías de la información con el desarrollo institucional, con propuestas para elevar la calidad de vida de la sociedad que la rodea.

CONCLUSIONES Los problemas de la universidad peruana, validados en el caso de estudio de la Escuela Profesional de Ingeniería Electrónica de la Universidad Nacional Pedro Ruiz Gallo de Lambayeque, Perú, el sector agroindustrial y su vínculo en la investigación, desarrollo tecnológico e innovación son: la universidad está aislada de la realidad que la rodea, escasa investigación de calidad, carencia de un sistema de medición, supervisión y control de las actividades de la universidad, cultura organizacional inadecua- da, carencia de competencias para la gestión universitaria en directivos, docentes y administrativos, escasos recursos para la formación profesional de calidad, y deficiente gestión de la información.

Las propuestas de solución se deben analizar y diseñar teniendo un enfoque que permita entender y resaltar la importancia de las relaciones con el entorno y contar con herramientas que logren manejar las nuevas variables para la gestión exitosa en estos tiempos de tecnologías de la información, globalización, calidad y medioambiente, y mercados muy competitivos. Se han comprobado las características pertinentes de la cibernética organizacional y en especial del modelo de los sistemas viables de Stafford Beer.

Las perspectivas de la Universidad Peruana son convertirse en una universidad de calidad, con investigaciones que contribuyan a la competitividad de las empresas y a la sostenibilidad del planeta, con una orientación a elevar la calidad de vida de la sociedad en la que está inmersa, decidida a lograr el alineamiento estratégico de las tecnologías de la información con el desarrollo institucional.

\section{REFERENCIAS BIBLIOGRÁFICAS}

ANR (2011). Datos estadísticos universitarios - Asamblea Nacional de Rectores, recuperado de: http:// censos.inei.gob.pe/cenaun/redatam_inei/doc/ ESTADISTICA_UNIVERSITARIAS.pdf

Beer, S. (1979). The Heart of Enterprice. Chichester: John Wiley \& Sons.

Beer, S. (1981). Brain of the Firm. Chichester: John Wiley \& Sons. Segunda edición.

Beer, S. (1985). Diagnosing the system for Organization. Chichester: John Wiley \& Sons.

I.E.E. Honorio Delgado (2013). Organización política y administrativa del territorio peruano, slide 3, recuperado de http://es.slideshare.net/ iehonoriodelgado/organizacin-poltica-yadministrativa-del-territorio-peruano

Ototsky, L. (2006). The Viable System Model. Recuperado de http://ototsky.mgn.ru/it/ papers/stafford21.pdf

Pérez Ríos, J. (2008). Diseño y Diagnóstico de Organizaciones Viables, Un enfoque sistémico. Valladolid: Publidisa.

Quispe Rojas, J. (2011). Sistematización de las Innovaciones de Empresas Industriales de Lambayeque de 2008 -2009. Revista Ciencia, Tecnología y Humanidades, 2 (2), 105 - 112.

(*) En Julio de 2014, se promulgó la nueva Ley Universitaria No. 30220. 